\title{
Use of Milnacipran in a Patient with Hyponatremia under the Cover of Fludrocortisones
}

\section{Sandeep Grover ${ }^{1 *}$, Mansi Somaiya ${ }^{2}$ and Pinaki Dutta ${ }^{3}$}

${ }^{1}$ Department of Psychiatry, Postgraduate Institute of Medical Education \& Research, Chandigarh 160012, India

${ }^{2}$ BYL Nair Ch. Hospital \& T. N. Medical College, Mumbai, India

${ }^{3}$ Department of Endocrinology, Postgraduate Institute of Medical Education \& Research, Chandigarh 160012, India

\begin{abstract}
Introduction: Hyponatremia is a known side effect of antidepressant medications. Usually management of hyponatremia in patients receiving antidepressants involves stopping of antidepressant and restriction of fluid intake. However, information is not clear with respect to use of antidepressant in patients with persistent hyponatremia.

Objective: To describe a patient detected to have hyponatremia prior to starting of antidepressant. The hyponatremia was corrected with the use of fludrocortisone, but introduction of sertraline led to reemergence of hyponatremia. Later patient tolerated milnacipran along with fludrocortisone, without recurrence of hyponatremia.

Conclusion: Fludrocortisone can be used for prevention and management of hyponatremia in patients at high risk of developing the same. Further, milnacipran may be considered as a viable option in patients who develop hyponatremia with SSRIs.
\end{abstract}

Keywords: Hyponatremia; Antidepressants; Fludrocortisone

\section{Introduction}

Hyponatremia (clinically defined as serum sodium levels of $<135$ $\mathrm{meq} / \mathrm{L}$ ) is considered to be the most common electrolyte abnormality seen in general hospital patients [1]. Hyponatremia is noted as a side effect associated with various psychotropic medications, especially the antidepressants. The risk of hyponatremia varies from one antidepressant to other. Evidence suggests that compared to other antidepressants the risk of hyponatremia is significantly greater with Selective Serotonin Reuptake Inhibitors (SSRIs) and Serotonin Norepinephrine Reuptake Inhibitors (SNRIs) [1-5]. Depending on the study design the incidence of SSRIs induced hyponatremia varies from 0.5 to $32 \%$ of the SSRIsand that for SNRIs is reported to be $4.2 \%$ [5,6]. Various risk factors for antidepressant induced hyponatremia include increasing age, female gender, presence of comorbid conditions like renal failure or other illnesses like hypertension, diabetes mellitus which can damage the kidneys, cardiac failure, volume depletion, hormonal imbalance and malignancies [1,3-5]. Further, hyponatremia with antidepressants is usually seen during the initial part of the therapy [4] and due to this some authors suggest that serum sodium levels must be monitored closely in patients who are at high risk of developing hyponatremia.

Usually, whenever a patient develops hyponatremia with an antidepressant, the usual management involves discontinuation of the antidepressant medication and strict fluid restriction. In rare patient, intravenous infusion of hypertonic saline may be required [7].

Data also suggests that rechallenge with the same or different antidepressants at times leads to recurrence of hyponatremia [8-10]. However, occasional reports suggest successful use of antidepressant like bupropion in patients who develop hyponatremia with sertraline [11]. Other reports suggest use of other strategies like tolvaptan [12].

Fludrocortisone is a synthetic corticosteroid which is known to have mineralocorticoid potency. No available report suggests use of fludrocortisone for management of antidepressant associated hyponatremia. In this case report we present a patient, detected to have hyponatremia prior to start of antidepressants, which was treated with fludrocortisone. However, while receiving fludrocortisone the patient had recurrence of hyponatremia with sertraline, but was able to tolerate milnacipran.

\section{Case Description}

Mrs A, 63 year old female, who was diagnosed with Diabetes Mellitus and hypertension 3 years back and hypothyroidism 2 years back, presented with Recurrent depressive disorder, current episode moderate depression without somatic symptoms. The current episode of depression was of 2 months duration. She was receiving Tab sitagliptin $100 \mathrm{mg} /$ day, Tab telmisartan $40 \mathrm{mg}$ /day, Tab amlodipine 5 $\mathrm{mg} /$ day, Tab ecosprin $75 \mathrm{mg} /$ day, Tab atorvastatin $10 \mathrm{mg} /$ day and tab thyronorm $75 \mathrm{mg} /$ day. In view of the age and physical comorbidity she was investigated before starting antidepressants and detected to have hyponatremia (serum sodium level-121 meq/L). In view of the same, antidepressant medication was not started and initially she was managed with tab clonazepam for anxiety and insomnia. She was referred to the endocrinologist, who on clinical and laboratory investigation could not find any specific cause for hyponatremia. She was advised for fluid restriction and to increase her salt intake. She was repeatedly investigated, over the period of 2 weeks, and was found to have persistent hyponatremia. Following this she was started on Tab fludrocortisone 50 microgram/day, with which within 1 week her serum sodium levels normalized ( $137 \mathrm{meq} / \mathrm{L})$. Following normalization of serum sodium levels, she was started on Tab sertraline $50 \mathrm{mg} /$ day. However, repeat serum sodium levels after 3 days revealed reduction in serum sodium levels $(130 \mathrm{meq} / \mathrm{L})$, despite being on fludrocortisone 50 microgram/day. Following this Tab sertraline was stopped and she was admitted in psychiatry inpatient unit due to worsening of depression. When reassessed in the inpatient unit, she was diagnosed with severe depression without psychotic symptoms (Hamilton depression rating

*Corresponding author: Sandeep Grover, Department of Psychiatry, Postgraduate Institute of Medical Education \& Research, Chandigarh 160012 India, Tel: 0091-172-2756807; E-mail: drsandeepg2002@yahoo.com

Received July 29, 2013; Accepted October 28, 2013; Published October 31 2013

Citation: Grover S, Somaiya M, Dutta P (2013) Use of Milnacipran in a Patient with Hyponatremia under the Cover of Fludrocortisones. J Clin Case Rep 3: 308. doi:10.4172/2165-7920.1000308

Copyright: $\odot 2013$ Grover S, et al. This is an open-access article distributed under the terms of the Creative Commons Attribution License, which permits unrestricted use, distribution, and reproduction in any medium, provided the original author and source are credited. 
scale total score-30). She was investigated and her routine investigations in the form of heamogram, liver function test, renal function test, and electrocardiogram did not reveal any abnormality. Her fasting glucose level was $147 \mathrm{mg} / \mathrm{dl}$ and post prandial glucose level was 197 $\mathrm{meq} / \mathrm{dl}$. No obvious cause could be detected for hyponatremia, other than sertraline. Her serum sodium level normalized to $145 \mathrm{meq} / \mathrm{L}$, 4 days after stopping sertraline. Following this in liaison with the endocrinologist, she was started on Inj Insulin (30:70), 24 units before breakfast and 10 units before dinner along with tab metformin $500 \mathrm{mg} /$ day after dinner. She was continued on Tab sitagliptin $100 \mathrm{mg} /$ day, Tab telmisartan $40 \mathrm{mg} /$ day, Tab amlodipine $5 \mathrm{mg} /$ day, Tab ecosprin $75 \mathrm{mg} /$ day, Tab atorvastatin $10 \mathrm{mg} /$ day and Tab thyroxine 75 microgram/day. Along with this she was given nutritional supplements in the form of folic acid, methylcobalamin and calcium along with tab clonazepam 0.5 $\mathrm{mg} /$ day twice daily. She was offered electroconvulsive therapy, but she refused.

After stopping sertraline and normalization of serum sodium levels, serum sodium levels were monitored for next 1 week, during which consistently the serum sodium levels were in the range of 140$145 \mathrm{meq} / \mathrm{L}$. Following this Tab milnacipran was introduced in the dose of $12.5 \mathrm{mg} /$ day along with continuation of Tab fludrocortisone 50 microgram/day along with monitoring of serum sodium. Gradually Tab milnacipran was increased to upto $125 \mathrm{mg}$ /day over the period of 3 weeks with close monitoring of serum sodium levels. During this period her serum sodium levels were always within the normal range 138-145 meq/L. With this treatment her depressive symptoms subsided and at the end of 5 week of inpatient stay her Hamilton Depression Rating Scale score reduced to 7. She followed up regularly on this treatment and maintained well for next 1 year. Her diabetes mellitus was also under control. Tab Fludrocortisone was stopped after 3 months of starting milnacipran.

\section{Discussion}

The present case highlights certain important issues. First, before prescribing antidepressants in persons with various risk factors to develop hyponatremia, it is worthwhile to get the baseline serum sodium levels, because many patients may have undetected hyponatremia, which may worsen with prescription of antidepressants. Second, the case highlights the fact that hyponatremia develops very early in the course of antidepressant therapy in certain vulnerable persons. Third, the case shows that fludrocortisone can be good therapeutic agent for management of hyponatremia in patients who do not respond to fluid restriction. Fourth, the case highlights the fact that antidepressant like milnacipran can be given to patients under the cover of fludrocortisone. Fifth, the case shows that even in the presence of fludrocortisone, patients may develop hyponatremia with sertraline, but not with milnacipran. This possibly reflects the fact that SSRIs have higher potential to cause hyponatremia, as reported in earlier reports [4].

Taking these facts into consideration, it can be said that high risk patients must be evaluated for hyponatremia at the base line and patients who develop hyponatremia with antidepressants, fluid restriction must be attempted along with stoppage of antidepressant as the first line management. However, when this does not work, use of fludrocortisone may be a considered as a viable option. In patients in whom use of antidepressants is a must, these may be used along with fludrocortisone along with monitoring of serum sodium levels. Further, milnacipran may be considered as a viable option in patients who develop hyponatremia with SSRIs.

\section{References}

1. Palmer BF, Gates JR, Lader M (2003) Causes and management of hyponatremia. Ann Pharmacother 37: 1694-1702.

2. Bogunovic OJ, Sotelo J, Madhusoodanan S (2003) Hyponatremia secondary to antidepressants. Psychiatric Annals 33: 333-339.

3. Movig KL, Leufkens HG, Lenderink AW, van den Akker VG, Hodiamont PP, et al. (2002) Association between antidepressant drug use and hyponatraemia: a case-control study. Br J Clin Pharmacol 53: 363-369.

4. Giorlando F, Teister J, Dodd S, Udina M, Berk M (2013) Hyponatraemia: an audit of aged psychiatry patients taking SSRIs and SNRIs. Curr Drug Saf 8: 175-180.

5. Jung YE, Jun TY, Kim KS, Bahk WM (2011) Hyponatremia associated with selective serotonin reuptake inhibitors, mirtazapine, and venlafaxine in Korean patients with major depressive disorder. Int J Clin Pharmacol Ther 49: 437-443.

6. Jacob S, Spinler SA (2006) Hyponatremia associated with selective serotoninreuptake inhibitors in older adults. Ann Pharmacother 40: 1618-1622.

7. Sharma H, Pompei $P$ (1996) Antidepressant-induced hyponatraemia in the aged. Avoidance and management strategies. Drugs Aging 8: 430-435.

8. Tsai PH, Chen HC, Liao SC, Tseng MC, Lee MB (2012) Recurrent escitalopraminduced hyponatremia in an elderly woman with dementia with Lewy bodies. Gen Hosp Psychiatry 34: 101.e5-7.

9. Stovall R, Brahm NC, Crosby KM (2009) Recurrent episodes of serotoninreuptake inhibitor-mediated hyponatremia in an elderly patient. Consult Pharm 24: 765-768.

10. Dirks AC, van Hyfte DM (2007) Recurrent hyponatremia after substitution of citalopram with duloxetine. J Clin Psychopharmacol 27: 313.

11. Cerimele JM, Robinson LA (2011) Sertraline-associated hyponatremia and subsequent tolerability of bupropion in an elderly woman. Prim Care Companion CNS Disord 13.

12. Meyer I, Frank D, Janssens U (2012) [A case of venlafaxine-induced syndrome of inappropriate $\mathrm{ADH}$ secretion (SIADH)-treatment with tolvaptan]. Dtsch Med Wochenschr 137: 1096-1099. 